\title{
CARBON NANOSTRUCTURED FILMS MODIFIED BY METAL NANOPARTICLES SUPPORTED ON FILTERING MEMBRANES FOR ELECTROANALYSIS
}

Erica Paramo, Susana Palmero*, Aranzazu Heras and Alvaro Colina.

Department of Chemistry, Universidad de Burgos, Pza Misael Bañuelos s/n, E-09001 Burgos, (SPAIN).

*Corresponding author: e-mail: spaldi@ubu.es.; Tel.: +34 947258817.

\begin{abstract}
A novel methodology to prepare sensors based on carbon nanostructures electrodes modified by metal nanoparticles is proposed. As a proof of concept, a novel bismuth nanoparticle/carbon nanofiber (Bi-NP/CNF) electrode and a carbon nanotube (CNT)/gold nanoparticle (Au-NP) have been developed. Bi-NP/CNF films were prepared by 1) filtering a dispersion of CNFs on a polytetrafluorethylene (PTFE) filter, and 2) filtering a dispersion of Bi-NPs chemically synthesized through this CNF/PTFE film. Next the electrode is prepared by sticking the $\mathrm{Bi}-\mathrm{NP} / \mathrm{CNF} / \mathrm{PTFE}$ film on a PET substrate. In this work, Bi-NP/CNF ratio was optimized using a $\mathrm{Cd}^{2+}$ solution as a probe sample. The $\mathrm{Cd}$ anodic stripping peak intensity, registered by differential pulse anodic stripping voltammetry (DPASV), is selected as target signal. The voltammograms registered for Cd stripping with this $\mathrm{Bi}-\mathrm{NP} / \mathrm{CNF} / \mathrm{PTFE}$ electrode showed well-defined and highly reproducible electrochemical. The optimized Bi-NP/CNF
\end{abstract}


electrode exhibits a $\mathrm{Cd}^{2+}$ detection limit of $53.57 \mathrm{ppb}$. To demonstrate the utility and versatility of this methodology, single walled carbon nanotubes (SWCNTs) and gold nanoparticles (Au-NPs) were selected to prepare a completely different electrode. Thus, the new Au-NP/SWCNT/PTFE electrode was tested with a multiresponse technique. In this case, UV/Vis absorption spectroelectrochemistry experiments were carried out for studying dopamine, demonstrating the good performance of the Au-NP/SWCNT electrode developed.

Keywords: Carbon nanofibers electrodes; Single-walled carbon nanotubes electrodes; Bismuth nanoparticles; Gold nanoparticles; Electroanalysis.

\section{Introduction}

Nanoparticles (NPs) modified electrodes are widely used in electroanalysis, and can be prepared using different methods. Carbon nanostructures such as carbon nanofibers (CNF) or carbon nanotubes (CNT) have been proposed as excellent materials to develop electrodes for analytical purposes [1-3]. The proper combination of these two different nanostructures, CNF or CNT and NPs, have led to very interesting sensors with applications in electrochemical biosensors, biomolecules and pharmaceuticals compounds [4-6]. The methods usually employed to prepare these hybrid substrates are drop-casting or spin-coating of chemically prepared NPs and/or CNF or CNT, or by direct electrodeposition of the NPs on the CNF or CNT modified electrode surface [4-7]. In this work, we proposed a new methodology based on our previously reported electrodes prepared on filtering membranes [8,9]. The main advantages of these electrodes are the simplicity of the process, since it does not involve the use of much more expensive techniques and reagents and it implies a greater reproducibility and homogeneity in the surface of the electrode. 
As a proof of concept, we have selected bismuth nanoparticles (Bi-NPs) as modifier of CNF electrodes supported on polytetrafluorethylene (PTFE) filters. In the last years, bismuth electrodes have been proposed as an alternative to mercury in stripping voltammetry [10-14] due to its low toxicity and wide electrochemical window [15-17]. Usually, Bismuth Film Electrodes (BiFEs) are obtained by depositing a bismuth layer on a suitable substrate such as graphite, carbon paste, glassy carbon, carbon nanofibers or graphene [18-22], using electrochemical methods such as voltammetry, pulse amperometry or galvanostatic methods. Other possibilities explored to prepare Bi modified electrodes implies the modification of carbon electrodes with bismuth oxide [6,16-17] or Bi-NPs [23-28]. Different solutions have been proposed to circumvent these drawbacks of direct electrodeposition of bismuth, such as screen printing a $\mathrm{Bi}$ nanopowder onto a conductive carbon layer [10] or drop-casting of $\mathrm{Bi}$ NPs on the electrode surface $[29,30]$.

In this work, a compact $\mathrm{Bi} / \mathrm{CNF}$ s/PTFE electrode is prepared by filtering a $\mathrm{Bi}-\mathrm{NP} /$ ethanol dispersion, chemically synthesized, through a CNFs/PTFE membrane that is directly used as working electrode in the electrochemical detection of $\mathrm{Cd}$ after fixing it onto a polyethyleneterephtalate (PET) substrate.

The new methodology used to prepare metal nanoparticle/carbon nanomaterial electrodes cab can be used with different combinations of these two nanomaterials. In order to demonstrate the utility and versatility we have selected a completely different combination of nanomaterials. In this case, a hybrid composite electrode formed by single-walled carbon nanotubes (SWCNTs) and gold nanoparticles (Au-NPs) have been prepared. This type of electrodes is receiving considerable attention because of the strong electrocatalytic activity $[31,32]$. The good performance of the new electrode (AuNPs/SWCNTs) was demonstrated by performing UV/Vis absorption spectroelectrochemistry of a well-known molecule, dopamine, typically determined with this type of electrocatalytic material. 
One of the main advantages of this methodology is the preparation of nanocomposite electrodes with the catalyst homogeneously distributed on the surface of the nanocarbon electrode and exposed to the solution to be analyzed.

The main objective of this work is to demonstrate the easiness of the new methodology and the good performance of the nanocomposite electrodes that can be used in any laboratory, for different purposes, using commercial nanomaterials, and avoiding the use of complex operations to obtain a good composite electrode.

\section{Material and methods}

\subsection{Materials}

Ethanol (Merck), polytetrafluoroethylene filters (PTFE) (pore size $0.1 \mu \mathrm{m}$; JVWP01300, Millipore Omnipore), $175 \mu \mathrm{m}$ thick sheets of polyethylene terephthalate (PET, HiFi Industrial Film), silver conductive paint (Electrolube) for the electrical contacts, and epoxy protective paint (242-SB de ESL Europe) as insulator, were used for the preparation of CNFs electrodes. CNFs nanofibers were vapour grown graphitized carbon fibers (VGCF®) from Showa Denko and SWCNTs nanotubes were commercial (Sigma-Aldrich).

Bi-NPs were chemically synthesized using $\mathrm{Bi}\left(\mathrm{NO}_{3}\right)_{3} \cdot 5 \mathrm{H}_{2} \mathrm{O}$ (Acros Organics) and $\mathrm{NaBH}_{4}$ (Acros Organics), and washed with acetone (Cofarcas). Au-NPs were synthesized from $\mathrm{HAuCl}_{4} \cdot 3 \mathrm{H}_{2} \mathrm{O}$ (Sigma-Aldrich) and tri-sodium citrate dyhidrate (Merck).

Cadmium standard solutions were prepared by dilution of a cadmium standard solution of $1000 \mathrm{mg} \mathrm{L}^{-1}$ (Merck) in a buffer solution $0.1 \mathrm{M}$ of acetic acid/sodium acetate (HAc/Ac', Merck), $\mathrm{pH}=4.5$. The solution $\mathrm{pHs}$ were measured with a $\mathrm{pH}$-meter (micropH 2002, Crison). Dopamine solutions (Acros Organics) were prepared in $0.1 \mathrm{M} \mathrm{HClO}_{4}$ (Acros Organics). 
All chemicals were analytical grade. Aqueous solutions were prepared using high-quality water (resistivity of $18.2 \mathrm{M} \Omega \cdot \mathrm{cm}$, MilliQ gradient A10 system, Millipore). All experiments were performed at room temperature.

\subsection{Instrumentation}

Electrochemical experiments using the BiNP/CNF/PTFE membrane as working electrode (WE) were carried out with a $\mathrm{CHI} 900$ (CH Instruments) potentiostat/galvanostat electrochemical system selecting a platinum wire as counter electrode (CE) and a home-made $\mathrm{Ag} / \mathrm{AgCl} / 3 \mathrm{M} \mathrm{KCl}$ microreference electrode (RE). All the experiments were performed in a drop of $100 \mu \mathrm{L}$ that was placed covering the WE. The CE and the RE electrode were placed in the drop on the WE.

Spectroelectrochemistry measurements were performed using a potentiostat/galvanostat (PGSTAT302N, Metrohm Autolab), a halogen-deuterium light source (AvaLight-DH-S-BAL, Avantes), a spectrometer (QE65000 198-1006 nm, Ocean Optics) and a reflection probe (FCR-7UV200, Avantes).

\subsection{Synthesis and characterization of bismuth nanoparticles}

Bi-NPs were synthetized modifying the procedure described by Van der Horst [25] where $\mathrm{Bi}^{3+} 0.01 \mathrm{M}$ was reduced by sodium borohydride $0.015 \mathrm{M}$ in aerobic conditions. Aggregated Bi-NPs were vacuum-filtered using a nitrocellulose filter (pore size $0.45 \mu \mathrm{m}, \mathrm{HAWPO} 2500$, Millipore Omnipore) and thoroughly washed with deionized water to remove impurities. Next, Bi-NPs were removed from the filter, washed with acetone and dried overnight at room temperature. Finally, Bi-NPs were dispersed in ethanol using a tip-sonicator (Optic ivymen System CY-500), and Bi-NPs solution with a concentration of $0.3 \mathrm{mg} / \mathrm{mL}$ was prepared. 
These NPs were characterized with transmission electron microscopy (TEM, JEOL JEM$1011 \mathrm{HR})$. Bi-NP samples for imaging were prepared by drop-casting on a TEM grid. The image processing software package ImageJ was used to quantify particle/grain size and size distribution. Fig. 1 shows the good dispersion of Bi-NPs that have a medium size of $3.6 \pm 0.3$ nm. A size lower than $4 \mathrm{~nm}$ is obtained for approx. a $70 \%$ of the NPs.

\subsection{Synthesis and characterization of gold nanoparticles}

Au-NPs were synthesized by Turkevich method [33], where $2 \mathrm{ml}$ of $0.038 \mathrm{M}$ sodium citrate were added to a $20 \mathrm{ml}$ of a heated up to boil chlorauric acid solution $\left(1 \cdot 10^{-3} \mathrm{M}\right.$ $\mathrm{HAuCl}_{4} \cdot 3 \mathrm{H}_{2} \mathrm{O}$ ). Then, the reaction mixture was stirred and boiled for 15 minutes, obtaining a stable red solution. The final UV-Vis spectrum of the nanoparticle dispersion is shown in Fig. 2. Showing the typical plasmon band at $521 \mathrm{~nm}$, where a narrow plasmon band centered is observed, indicating that monodispersed Au-NPs were synthesized. SEM images were obtained on the nanocomposite electrode, as will be shown and discussed below.

\subsection{Bi-NP/CNF Electrode fabrication}

A solution of CNFs $0.1 \mathrm{mg} / \mathrm{mL}$ was dispersed in ethanol by ultrasonication using a tipsonicator (Optic ivymen System CY-500) for 60 minutes. A known volume of the CNFs dispersion was vacuum-filtered using a $0.1 \mu \mathrm{m}$-diameter PTFE filter (Merck-Millipore). Next, a known volume of the $0.3 \mathrm{mg} / \mathrm{mL}$ Bi-NPs dispersion in ethanol was vacuum-filtered through the CNF/PTFE membrane. The obtained hybrid material was dried for 12 hours under room temperature. The Bi-NP/CNF/PTFE film was fixed to a PET sheet with an inert epoxy insulating paint (Fig 3A). Electrical contacts were made using the conductive silver paint that was dried in an oven at $70{ }^{\circ} \mathrm{C}$ for 40 minutes. Next, electrical contacts were electrically isolated using the inert epoxy insulating paint (Fig 3B). Finally, a Teflon mask was fixed to the $\mathrm{Bi}-\mathrm{NP} / \mathrm{CNF} / \mathrm{PTFE}$ film to limit and control the geometrical area of the Bi-NP/CNF film 
(Fig. 3C). The scanning electron microscopy (SEM) image (FEI - Quanta 200FEG) of the BiNP/CNF/PTFE film is shown in Fig. 4, showing that CNFs are fully covered with Bi-NPs.

Thickness of the CNFs film was determined by Atomic-force microscopy (AFM) ( Confocal Raman Microscope - Alpha300R - Alpha300A AFM Witec), obtaining a value of 15,5 $\pm 0,2$ $\mu \mathrm{m}$.

\subsection{Au-NP/SWCNT Electrode fabrication}

AuNP/SWCNT electrodes were made following the next steps. First, a SWCNT dispersion $0.005 \mathrm{mg} / \mathrm{mL}$ in 1,2-dicloroetane (DCE, Acros Organics) was prepared. To get an appropriate homogenization, the dispersion was sonicated for 3 minutes using a tip-sonicator. Then, 1.5 $\mathrm{mL}$ of SWCNT dispersion was vacuum-filtered through a hydrophilic $0.1 \mu \mathrm{m}$-diameter PTFE filter (Merck-Millipore) obtaining a SWCNT homogeneous film that was dried under nitrogen current. Next, $1.5 \mathrm{~mL}$ of Au-NP dispersion were vacuum-filtered through the SWCNT/PTFE membrane, obtaining a homogenous film of Au-NPs deposited on the CNTs film. The obtained hybrid material was dried for 12 hours under room temperature.

Afterwards, the AuNP/SWCNT/PTFE membrane was fixed on a clean PET sheet with the inert epoxy insulating paint applied in the external section of the filter. The electrical contact was made using the conductive silver paint that was dried in an oven at $70{ }^{\circ} \mathrm{C}$ for 40 minutes. Then, part of this silver electrical contact and the AuNPs/CNTs/PTFE filter was electrically isolated and delimited with a thin layer of kapton.

Thickness of the CNTs film was determined by Atomic-force microscopy (AFM)( Confocal Raman Microscope - Alpha300R - Alpha300A AFM Witec), obtaining a value of $435 \pm 70$ nm. 
AuNP/CNT/PTFE membrane was characterized with scanning electron microscopy (ZEISS, Merlin VP Compact). Fig 5A shows the SEM image of CNTs after filtering through the PTFE filter and Fig 5B shows the distribution of AuNPs over CNTs/PTFE after filtering. The AuNPs synthesized has a medium size of $41.5 \pm 0.6 \mathrm{~nm}$.

\subsection{Electrochemical impedance spectroscopy (EIS)}

Electrochemical impedance spectroscopy (EIS) was carried out with Autolab PGSTAT 30 Potentiostat/Galvanostat system (Eco Chemie, the Netherlands) with frequency response analyzer (FRA). The scanning frequency ranged from $5 \bullet 104$ to $1.5 \mathrm{~Hz}$ and amplitude $0.01 \mathrm{~V}$.

Fig.6. shows the results of EIS for both electrodes, CNTs and CNFs for a equimolar concentration $10 \mathrm{mM}\left[\mathrm{Fe}(\mathrm{CN})_{6}\right]^{3-/ 4-}$ in $1 \mathrm{M} \mathrm{KNO}_{3}$. The Faradaic impedance spectra, presented as Nyquist plots. The CNTs Nyquist plot (Fig.6a) exhibited almost a straight line with a small semicircle arc $(\mathrm{R}=81.6 \Omega)$ represents the characteristics of diffusion limited electron-transfer process on the electrode surface. On the same conditions, CNFs Nyquist plot (Fig.6b) shows a small deformation on the nyquist plot $(\mathrm{R}=894 \Omega)$ due to the film thickness and the more hydrophilicity carbon nanofiber presents which increases the energy barrier for the ion transfer between the coating and the electrolyte exhibited a thin layer o adsorption phenomena and it is possible to obtain an estimate of the CNFs film resistance.

\section{Results and Discussion}

\subsection{Parameters optimization for the fabrication of the Bi-NP/CNF electrodes}

Experimental design was used to optimize the most influential parameters in the fabrication of the Bi-NP/CNF/PTFE electrode: the amount of CNF dispersion and the amount of Bi-NP dispersion in ethanol that should be filtered. A circumscribed central composite 
design for two factors was selected to optimize these two parameters, thus, performing only 11 experiments, the best conditions to prepare the Bi-NP/CNF electrodes were obtained. The central point in the design (0.04 mg of CNFs, $0.33 \mathrm{mg}$ of Bi-NPs) was replicated three times to estimate the experimental error. Two levels, high (0.06 $\mathrm{mg}$ of CNFs, $0.48 \mathrm{mg}$ of Bi-NPs) and low (0.02 mg of CNF, $0.18 \mathrm{mg}$ of Bi-NPs), were chosen to carry out the experimental designs. For this central composite design, the highest values and the lowest values studied for the factors were $0.068 \mathrm{mg}$ of CNF, $0.54 \mathrm{mg}$ of Bi-NPs and $0.012 \mathrm{mg}$ of CNF, $0.12 \mathrm{mg}$ of Bi-NPs, respectively.

A $200 \mathrm{ppb} \mathrm{Cd}^{2+}$ solution in $\mathrm{HAc} / \mathrm{Ac}^{-}$buffer $(\mathrm{pH}=4.5)$ was selected to optimize the response of the analytical method. First, $\mathrm{Cd}^{2+}$ was reduced and accumulated on the electrode at $-1.40 \mathrm{~V}$ for $180 \mathrm{~s}$ and, next, the potential was scanned from $-0.85 \mathrm{~V}$ to $-0.60 \mathrm{~V}$, with a step increment of $5 \mathrm{mV}$, amplitude of $50 \mathrm{mV}$, pulse width of $0.25 \mathrm{~s}$ and pulse period of $0.65 \mathrm{~s}$, to re-oxidized the preconcentrated $\mathrm{Cd}$, avoiding the stripping of the Bi-NPs. The current intensity of the $\mathrm{Cd}^{2+}$ stripping peak was used as response variable in the experimental design. The surface response obtained from the design shows a maximum using $0.068 \mathrm{mg}$ of CNFs and $0.520 \mathrm{mg}$ of Bi-NPs, which were taken as the optimum values to fabricate the Bi-NP/CNF/PTFE WE as sensing platform of $\mathrm{Cd}^{2+}$. It is noteworthy that $\mathrm{mg}$ of CNF has not effect in the response (pvalue $>0.34$ ), but there is a clear effect of $\mathrm{mg}$ of Bi-NPs ( $\mathrm{p}$-value $<0.03$ ). These results indicate that the amount of CNF is high enough to produce electrodes with good conductivity that can be used for electroanalysis. The amount of Bi-NPs is important because the stripping signal of $\mathrm{Cd}$ is improved at high amounts of Bi-NPs, indicating that more $\mathrm{Cd}^{2+}$ can be reduced on the Bi-NPs. There is an interesting interaction between $\mathrm{mg}$ of CNFs and $\mathrm{mg}$ of Bi-NPs (pvalue $<0.02$ ) that indicates that low amounts of Bi-NPs exhibit good responses when the NPs are immobilized on electrodes formed by low amounts of CNFs. However, the best response 
is obtained for electrodes with a high amount of both CNFs and an intermediate amount of Bi-NPs.

\subsection{Cadmium detection with Bi-NP/CNF/PTFE WE}

Once the fabrication of the electrode was optimized, differential pulse anodic stripping voltammetry (DPASV) experiments were performed with a set of calibration samples with $\mathrm{Cd}^{2+}$ concentrations between 25 and $900 \mathrm{ppb}$ to demonstrate the usefulness of the $\mathrm{Bi}$ $\mathrm{NP} / \mathrm{CNF} / \mathrm{PTFE}$ electrodes for electroanalysis. This concentration range has been selected considering the maximum levels allowed in food according to the commission regulation (EC) No 1881/2006 [34]. Experimental conditions were the same established for the experimental design described above. Fig.7 shows the DPASV voltammograms registered during Cd stripping, where a relationship is observed between the peak current and the $\mathrm{Cd}$ concentration. After outlier detection by least median of squares regressions (LMS), the ordinary least square regression model (OLS) was performed obtaining the next results: $\Delta \mathrm{I}$ $(\mathrm{A})=1.83 \cdot 10^{-8} \cdot \mathrm{C}_{\mathrm{Cd}}{ }^{2+}(\mathrm{ppb})-2.99 \cdot 10^{-7} . \mathrm{A}$ good determination coefficient $\left(\mathrm{R}^{2}=0.999\right)$ and a low residual standard error $\left(\mathrm{S}_{\mathrm{yx}}=2.28 \cdot 10^{-7}\right)$ were obtained indicating the good quality of regression model constructed. The detection limit calculated with this linear model is 53.57 $\mathrm{ppb}$ of $\mathrm{Cd}^{2+}$.

The reproducibility of the fabrication method proposed to obtain this hybrid Bi-NP/CNF sensor was tested using the slopes of three sets of calibrations obtained with different $\mathrm{Bi}$ NP/CNF/PTFE electrodes under the same experimental conditions. The average slope ([1.83 $\left.\pm 0.08] \cdot 10^{-8} \mathrm{~A} \cdot \mathrm{ppb}^{-1}\right)$ and the low relative standard deviation $(\% \mathrm{RSD}=2.5 \%, \mathrm{n}=3)$ of these regressions demonstrates the good reproducibility of the methodology proposed to prepare BiNP modified electrodes. 
A test sample with a known concentration of $600 \mathrm{ppb}$ was prepared to prove the prediction capability of the analytical method developed using these new Bi-NP/CNF/PTFE electrodes. A predicted value of $617.1 \pm 30.7 \mathrm{ppb}$ was calculated with a low residual standard deviation $(\% \mathrm{RSD}=4.1 \%, \mathrm{n}=3)$, indicating the good performance of these electrodes to $\mathrm{Cd}$ detection.

\subsection{Spectroelectrochemical analysis of dopamine over AuNP/CNT/PTFE electrodes}

The previously described filtration system allowed the fabrication of electrodes with different carbon supports that can be modified with a range of NPs. A second WE were prepared following the procedure described above to obtain, in this case, a AuNP/SWCNT/PTFE electrode. These electrodes can be used for different analytical purposes. In this case, we have selected a UV/Vis absorption spectroelectrochemistry experiment to demonstrate the suitability of the composite as working electrode. A $10^{-3} \mathrm{M}$ dopamine (DA) solution in $0.1 \mathrm{M}$ $\mathrm{HClO}_{4}$ was selected to observe the behavior of the Au-NP/CNT/PTFE electrode. First, dopamine was oxidized while the potential was scanned from $+0.25 \mathrm{~V}$ to $+0.75 \mathrm{~V}$ with a scan rate of $0.005 \mathrm{~V} \mathrm{~s}^{-1}$ avoiding the stripping of the Au-NPs, and next, it was reduced in the cathodic scan. Fig.8a shows the cyclic voltammogram registered, where an oxidation peak emerges at $+0.55 \mathrm{~V}$ while a reduction peak is observed at $+0.51 \mathrm{~V}$ in the backward scan, displaying the reversibility of the system. Fig.8b shows the typical evolution of dopaminequinone UV-Vis spectral signal obtained during the electrochemical process, taking as reference spectrum that of the initial solution with dopamine before starting the voltammetric scan. Two absorption bands, peaking at $391 \mathrm{~nm}$ and $246 \mathrm{~nm}$, increase indicating that dopaminequinone has been generated, while a band at $289 \mathrm{~nm}$ decreases indicating the consumption of dopamine. Negative values of absorbance were observed because the initial solution is taken as reference spectrum. Fig. $8 \mathrm{c}$ shows the voltabsorptogram at the wavelength of the maximum absorbance, $391 \mathrm{~nm}$. Absorbance grows during the anodic scan and decreases during the cathodic one, demonstrating the reversibility of the redox couple on the 
$\mathrm{Au}-\mathrm{NP} / \mathrm{CNT} / \mathrm{PTFE}$ electrode. Evolution of absorbance at $246 \mathrm{~nm}$ is completely similar to 391 $\mathrm{nm}$, and the symmetric response is obtained at $289 \mathrm{~nm}$, because dopamine concentration decreases in the forward scan and is re-generated in the backward one. This experiment demonstrates the good performance of the electrode that has been fabricated from completely different nanomaterials, pointing out the good performance of the methodology to prepare diverse types of composites.

\section{Conclusions}

In this work, a new, easy and reproducible methodology to modified carbon nanostructured electrodes with metal NPs has been proposed. As a proof of concept, the viability of an electrode based on Bi-NPs and CNFs have been demonstrated. Experimental design has allowed us to optimize the preparation of modified electrodes. These Bi-NP/CNF/PTFE electrodes have been demonstrated to be very useful for the determination of $\mathrm{Cd}^{2+}$. This methodology allows us to fabricate easily hybrid electrodes with various nanostructures by selecting different carbon nanomaterials as support electrode material. Moreover, the utility and versatility of the new methodology to prepare composites with different NPs and nanocarbon materials has been demonstrated using AuNPs and SWCNTs with the spectroelectrochemical study of dopamine. The results presented in this work open up new avenues in electroanalytical determinations. Therefore, a number of future analytical applications for this type of electrodes can be expected.

\section{Acknowledgements}

We greatly thank Dra. Virginia Ruiz from CIDETEC (Spain) for supplying carbon nanofibers. Financial support from Ministerio de Economía y Competitividad (CTQ201461914-EXP，CTQ2014-55583-R，TEC2014-51940-C2-2R，CTQ2015-71955-REDT) and Junta de Castilla y León (BU033-U16) is gratefully acknowledged. 


\section{References}

[1] J. Huang, Y. Liu, T. You, Carbon nanofiber based electrochemical biosensors: A review, Anal. Methods. 2 (2010) 202-211. doi:10.1039/b9ay00312f.

[2] J. Wang, Y. Lin, Functionalized carbon nanotubes and nanofibers for biosensing applications, TrAC Trends Anal. Chem. 27 (2008) 619-626. doi:10.1016/j.trac.2008.05.009.

[3] E. James, Graphene, Carbon Nanotubes, and Nanostructures: Techniques and Applications, (2017), CRC Press, n.d.

[4] C. Yang, M.E. Denno, P. Pyakurel, B.J. Venton, Recent trends in carbon nanomaterial-based electrochemical sensors for biomolecules: A review, Anal. Chim. Acta. 887 (2015) 17-37. doi:10.1016/j.aca.2015.05.049.

[5] X. Ge, A.M. Asiri, D. Du, W. Wen, S. Wang, Y. Lin, Nanomaterial-enhanced paperbased biosensors, TrAC - Trends Anal. Chem. 58 (2014) 31-39. doi:10.1016/j.trac.2014.03.008.

[6] B.R. Adhikari, M. Govindhan, A. Chen, Carbon nanomaterials based electrochemical sensors/biosensors for the sensitive detection of pharmaceutical and biological compounds, Sensors. 15 (2015) 22490-22508. doi:10.3390/s150922490.

[7] G. Aragay, A. Merkoçi, Nanomaterials application in electrochemical detection of heavy metals, Electrochim. Acta. 84 (2012) 49-61. doi:10.1016/j.electacta.2012.04.044.

[8] J. Garoz-Ruiz, A. Heras, S. Palmero, A. Colina, Development of a Novel Bidimensional Spectroelectrochemistry Cell Using Transfer Single-Walled Carbon Nanotubes Films as Optically Transparent Electrodes, Anal. Chem. 87 (2015) 6233-6239. doi:10.1021/acs.analchem.5b00923.

[9] A.E. Paramo, S. Palmero, A. Heras, A. Colina, D. Ibañez, Development of Disposable Carbon Nanofibers Electrodes Supported on Filters, Electroanalysis. 28 (2016) 890-897. doi:10.1002/elan.201500576.

[10] G.-J. Lee, H.-M. Lee, C.-K. Rhee, Bismuth nano-powder electrode for trace analysis of heavy metals using anodic stripping voltammetry, Electrochem. Commun. 9 (2007) 25142518. doi:10.1016/j.elecom.2007.07.030.

[11] I. Rutyna, M. Korolczuk, Determination of cobalt by adsorptive stripping voltammetry with double accumulation and stripping steps, Int. J. Environ. Anal. Chem. (2015) 1-12. doi:10.1080/03067319.2015.1078799.

[12] M. Frena, I. Campestrini, O.C. de Braga, A. Spinelli, In situ bismuth-film electrode for square-wave anodic stripping voltammetric determination of tin in biodiesel, Electrochim. Acta. 56 (2011) 4678-4684. doi:10.1016/j.electacta.2011.02.111.

[13] B. Nigović, B. Šimunić, S. Hocevar, Voltammetric measurements of aminosalicylate drugs using bismuth film electrode, Electrochim. Acta. 54 (2009) 5678-5683. doi:10.1016/j.electacta.2009.05.006.

[14] A. Królicka, A. Bobrowski, Bismuth film electrode for adsorptive stripping voltammetry - electrochemical and microscopic study, Electrochem. Commun. 6 (2004) 99104. doi:10.1016/j.elecom.2003.10.025.

[15] I. Švancara, C. Prior, S.B.B. Hočevar, J. Wang, A Decade with Bismuth-Based Electrodes in Electroanalysis, Electroanalysis. 22 (2010) 1405-1420.

doi:10.1002/elan.200970017. 
[16] K. Vytřas, I. Švancara, R. Metelka, A novelty in potentiometric stripping analysis: total replacement of mercury by bismuth, Electroanalysis. 14 (2002) 1359-1364. doi:10.1002/1521-4109(200211)14:19/20<1359::AID-ELAN1359>3.0.CO;2-P.

[17] J. Wang, J. Lu, S.B. Hocevar, B. Ogorevc, B. Wang, J., Lu, J., Hocevar, S.B. Ogorevc, Bismuth-Coated Screen-Printed Electrodes for Stripping Voltammetric Measurements of Trace Lead, Electroanalysis. 13 (2001) 13-16. doi:10.1002/15214109(200101)13:1<13::AID-ELAN13>3.0.CO;2-F.

[18] M.A. Chamjangali, H. Kouhestani, F. Masdarolomoor, H. Daneshinejad, A voltammetric sensor based on the glassy carbon electrode modified with multi-walled carbon nanotube/poly(pyrocatechol violet)/bismuth film for determination of cadmium and lead as environmental pollutants, Sensors Actuators, B Chem. 216 (2015) 384-393. doi:10.1016/j.snb.2015.04.058.

[19] É.S. Sá, P.S. Da Silva, C.L. Jost, A. Spinelli, Electrochemical sensor based on bismuth-film electrode for voltammetric studies on vitamin B2 (riboflavin), Sensors Actuators, B Chem. 209 (2015) 423-430. doi:10.1016/j.snb.2014.11.136.

[20] J. Wang, Stripping Analysis at Bismuth Electrodes: A Review, Electroanalysis. 17 (2005) 1341-1346. doi:10.1002/elan.200403270.

[21] S. Lee, S. Bong, J. Ha, M. Kwak, S.K. Park, Y. Piao, Electrochemical deposition of bismuth on activated graphene-nafion composite for anodic stripping voltammetric determination of trace heavy metals, Sensors Actuators, B Chem. 215 (2015) 62-69. doi:10.1016/j.snb.2015.03.032.

[22] E. Tesarová, A. Heras, A. Colina, V. Ruiz, I. Svancara, K. Vytras, J. López-Palacios, A spectroelectrochemical approach to the electrodeposition of bismuth film electrodes and their use in stripping analysis., Anal. Chim. Acta. 608 (2008) 140-146. doi:10.1016/j.aca.2007.12.023.

[23] G.H. Hwang, W.K. Han, J.S. Park, S.G. Kang, Determination of trace metals by anodic stripping voltammetry using a bismuth-modified carbon nanotube electrode., Talanta. 76 (2008) 301-308. doi:10.1016/j.talanta.2008.02.039.

[24] R. Jain, R. Sharma, Novel bismuth/multi-walled carbon nanotubes-based electrochemical sensor for the determination of neuroprotective drug cilostazol, J. Appl. Electrochem. 42 (2012) 341-348. doi:10.1007/s10800-012-0402-8.

[25] N. A. Malakhova, A. A. Mysik, S.Y. Saraeva, N.Y. Stozhko, M. a. Uimin, A. E. Ermakov, K.Z. Brainina, A voltammetric sensor on the basis of bismuth nanoparticles prepared by the method of gas condensation, J. Anal. Chem. 65 (2010) 640-647. doi:10.1134/S1061934810060158.

[26] M.A.G. Rico, M. Olivares-Marín, E.P. Gil, Modification of carbon screen-printed electrodes by adsorption of chemically synthesized $\mathrm{Bi}$ nanoparticles for the voltammetric stripping detection of $\mathrm{Zn}(\mathrm{II}), \mathrm{Cd}(\mathrm{II})$ and $\mathrm{Pb}(\mathrm{II})$., Talanta. 80 (2009) 631-635. doi:10.1016/j.talanta.2009.07.039.

[27] V.B. Dos Santos, E.L. Fava, N.S. De Miranda Curi, R.C. Faria, O. Fatibello-Filho, A thermostated electrochemical flow cell with a coupled bismuth film electrode for square-wave anodic stripping voltammetric determination of cadmium(II) and lead(II) in natural, wastewater and tap water samples, Talanta. 126 (2014) 82-90. doi:10.1016/j.talanta.2014.03.015. 
[28] X. Chen, S. Chen, W. Huang, J. Zheng, Z. Li, Facile preparation of Bi nanoparticles by novel cathodic dispersion of bulk bismuth electrodes, Electrochim. Acta. 54 (2009) 73707373. doi:10.1016/j.electacta.2009.07.068.

[29] S.W. Jones, R.G. Compton, Fabrication and Applications of Nanoparticle-Modified Electrodes in Stripping Analysis., Curr. Anal. Chem. 4 (2008) 177-182. doi:10.2174/157341108784911370 .

[30] C. Van der Horst, B. Silwana, E. Iwuoha, V. Somerset, Synthesis and Characterization of Bismuth-Silver Nanoparticles for Electrochemical Sensor Applications, Anal. Lett. 48 (2014) 1311-1332. doi:10.1080/00032719.2014.979357.

[31] M.-P. Ngoc Bui, C.A. Li, K.N. Han, X.-H. Pham, G.H. Seong, Simultaneous detection of ultratrace lead and copper with gold nanoparticles patterned on carbon nanotube thin film, Analyst. 137 (2012) 1888-1894. doi:10.1039/c2an16020j.

[32] Z. Zhao, Y. Sun, P. Li, W. Zhang, K. Lian, J. Hu, Y. Chen, Preparation and characterization of AuNPs/CNTs-ErGO electrochemical sensors for highly sensitive detection of hydrazine, Talanta. 158 (2016) 283-291. doi:10.1016/j.talanta.2016.05.065.

[33] S. Kumar, K.S. Gandhi, R. Kumar, Modeling of Formation of Gold Nanoparticles by Citrate Method †, Ind. Eng. Chem. Res. 46 (2007) 3128-3136. doi:10.1021/ie060672j.

[34] T.H.E. Commission, O.F. The, E. Communities, 20.12.2006, (2006) 5-24. 


\section{Figure captions}

Fig. 1: TEM image and histogram for Bi-NPs.

Fig. 2: UV-Vis spectra of Au-NPs.

Fig. 3: Steps in electrode fabrication: Step A: CNF film, (1) PTFE filter (2) PET sheet (3) conductive silver paint (4). Step B: Insulation of conductive silver paint and contact between silver paint and CNF film with the inert epoxy insulating paint (5). Step C: Teflon mask to limit and control the geometrical area of the Bi-NP/CNF WE (6).

Fig. 4: SEM image of the Bi-NP/CNF/PTFE membrane.

Fig. 5: (a) SEM image of SWCNTs film on the PTFE filter. (b) SEM image of the AuNP/SWCNT/PTFE membrane.

Fig. 6. EIS Nyquist plots of electrochemical impedance spectroscopy (EIS) for a) $\mathrm{CNTs} / \mathrm{PTFE} / \mathrm{PET}$ electrode and b) CNFs/PTFE/PET electrode in the presence of equimolar $10 \mathrm{mM}\left[\mathrm{Fe}(\mathrm{CN})_{6}\right]^{3-/ 4-}$ in $1 \mathrm{M} \mathrm{KNO}_{3}$. Frequency ranges from $5 \cdot 104$ to $1.5 \mathrm{~Hz}$.

Fig. 7: DPASV voltammograms recorded during the Cd stripping after being preconcentrated applying $-1.40 \mathrm{~V}$ during $180 \mathrm{~s}$. $\mathrm{E}_{\text {initial }}=-0.85 \mathrm{~V}, \mathrm{E}_{\text {final }}=-0.60 \mathrm{~V}$, step increment of $5 \mathrm{mV}$, amplitude of $50 \mathrm{mV}$, pulse width of $0.25 \mathrm{~s}$ and pulse period of $0.2 \mathrm{~s} . \mathrm{C}_{\mathrm{Cd}}{ }^{2+}=25-900 \mathrm{ppb}$ in HAc/Ac ${ }^{-}$buffer $(\mathrm{pH}=4.5)$.

Fig. 8: (a) Cyclic voltammogram of dopamine $10^{-3} \mathrm{M}, \mathrm{E}_{\text {initial }}=+0.25 \mathrm{~V}, \mathrm{E}_{\text {final }}=+0.75 \mathrm{~V}$, scan rate $0.005 \mathrm{~V} \cdot \mathrm{s}^{-1}$. (b) $3 \mathrm{D}$ absorption spectra evolution of dopamine during the cyclic voltammetry. (c) Voltabsorptogram of dopamine at $391 \mathrm{~nm}$. 